\title{
WHAT DO WE KNOW ABOUT POSTOPERATIVE DELIRIUM (POD)? RESULTS OF A NATIONAL SURVEY
}

\author{
1- Centro Hospitalar do Porto, Departamento de Anestesiologia, Emergência e Cuidados Intensivos, Porto \\ 2- Centro de Investigação Clínica em Anestesiologia \\ 3- Centro Hospitalar de São João, Departamento de Anestesiologia, Porto, Portugal
}

Maria João Susano ${ }^{1,2}$, Liliana Vasconcelos ${ }^{1}$, Tiago Lemos ${ }^{1}$, Pedro Amorim,2, Fernando Abelha ${ }^{3}$

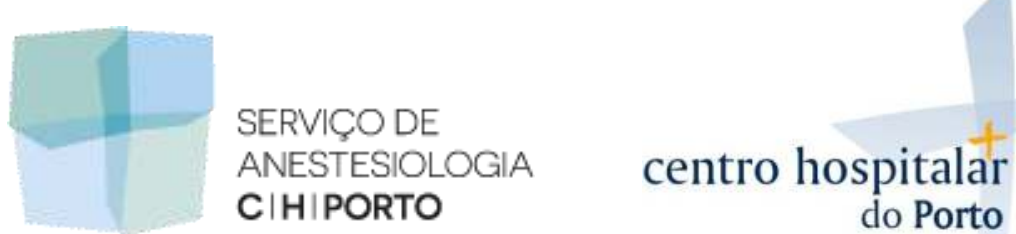

\section{BACKGROUND AND GOAL OF STUDY}

POD is the most common postoperative complication in elderlies, but highly under-diagnosed.

The aim of the study was to assess anaesthesiologists perspectives and management of POD.

\section{RESULTS}

\section{4 surveys}

Population description is presented in Image 1.

Detailed results are shown in Table 1.
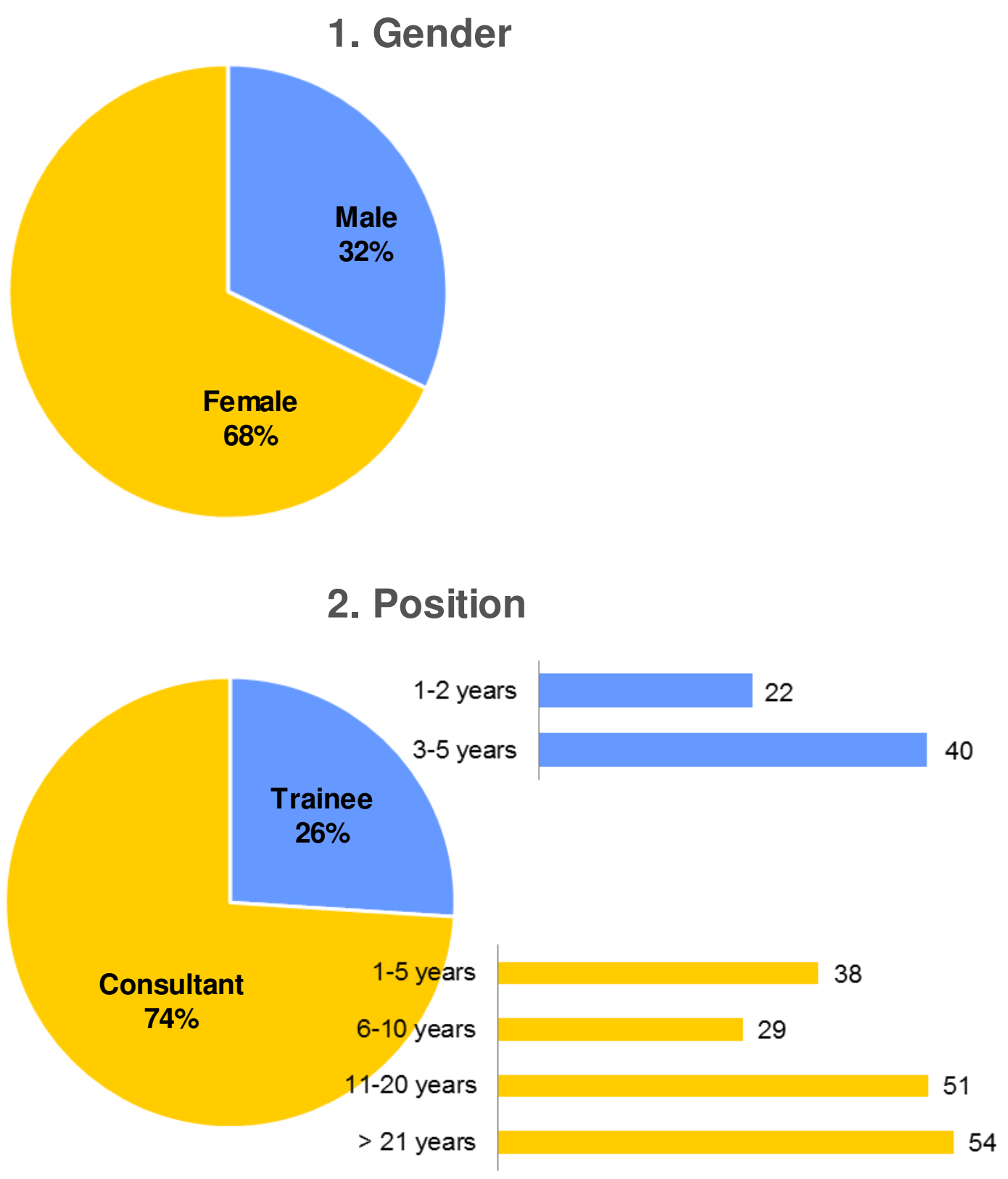

3. Teaching Hospital

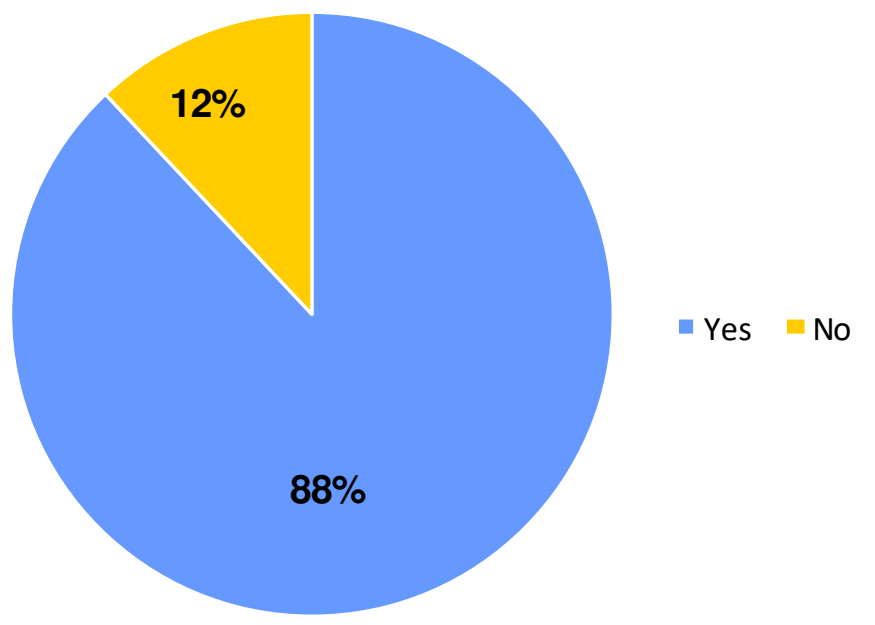

Image 1

\section{CONCLUSIONS}

Overall, anaesthesiologists are aware of the importance, risk factors and management for POD. Intraoperative monitoring of DA is recommended to lower the incidence of POD; although it is widely used there is room for improvement. Protocols for preoperative cognition evaluation and POD diagnosis and treatment should be sought.

\section{METHODS}

Target Population: Portuguese anaesthesiologists Online adapted survey (1) divided in 3 sections: -Demographic data;

-Preferences, routines and practices;

- Clinical cases.

Answers presented as a Likert scale from 1 to 5 or as yes/no/don't know

Descriptive statistical analysis

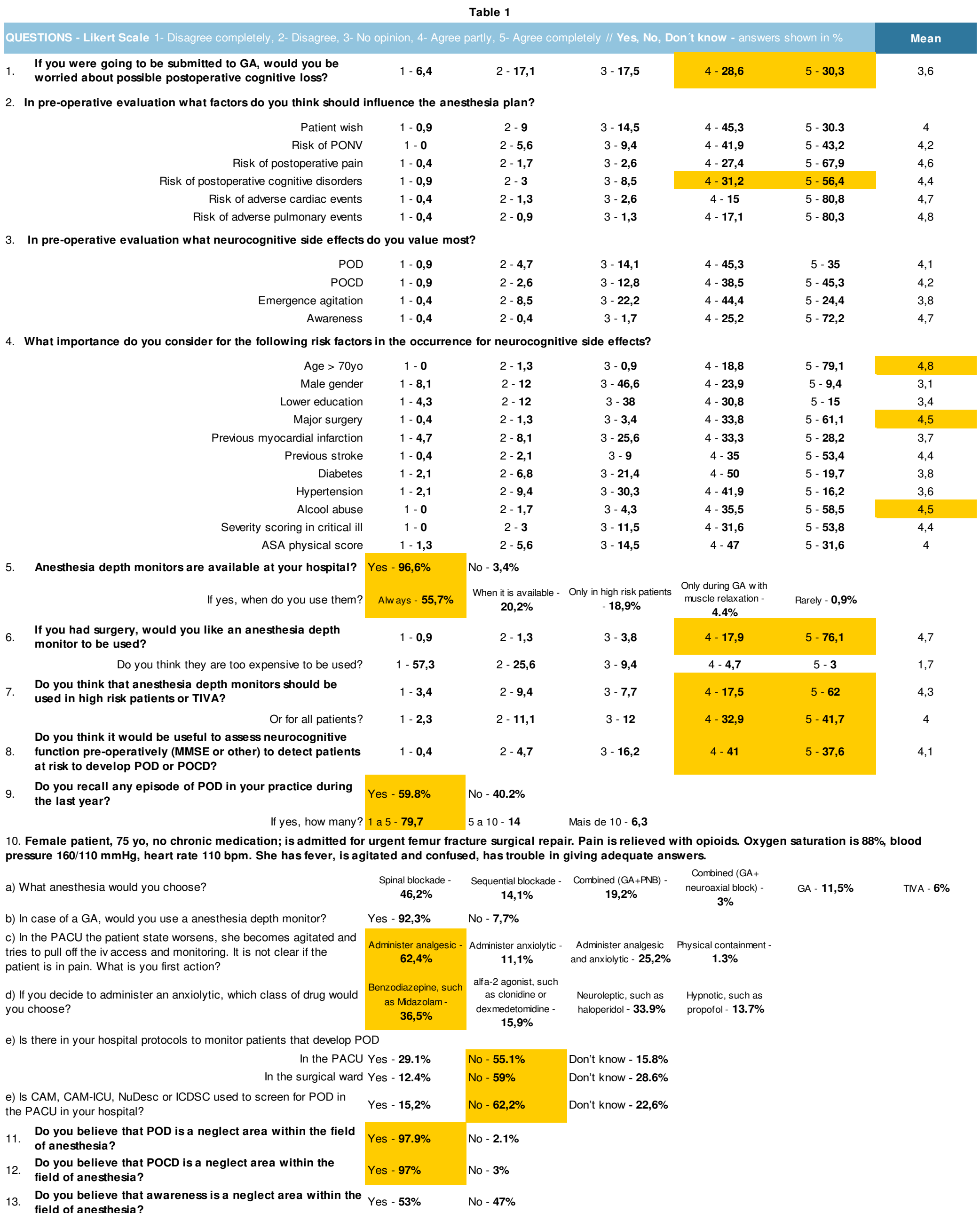

\section{Table 1: \%: percentage; GA: general anesthesia; PONV: postoperative nausea and vomit; POD: postoperative delirium; POCD: postoperative cognitive dysfunction; yo: years old; ASA:} American Society of Anaesthesiologists; TIVA: total intravenous anaesthesia; MMSE: Mini Mental State Examination; PNB: peripheral nerve block; PACU: Post Anesthesia Care Unil 